Gynecologic and

Obstetric Investigation
Gynecol Obstet Invest 2013;76:151-157

DOI: $10.1159 / 000351875$
Received: September 24, 2012

Accepted after revision: May 8, 2013

Published online: August 14, 2013

\title{
Management of Caesarean Scar Pregnancies Using an Intrauterine or Abdominal Approach Based on the Myometrial Thickness between the Gestational Mass and the Bladder Wall
}

\author{
Ming-Jun Shao Mei-Xu Hu Xiao-Jiu Xu Lin Zhang Min Hu \\ Department of Obstetrics and Gynecology, Jinhua Municipal Central Hospital, Jinhua, Zhejiang Province, PR China
}

\section{Key Words}

Caesarean scar pregnancy · Endoscopy · Methotrexate ·

Uterine artery embolization

\begin{abstract}
Aim: To describe our experience with various interventions for caesarean scar pregnancies (CSPs) based on the myometrial thickness between the gestational mass and the bladder. Methods: All patients were initially administered methotrexate. Then, the appropriate therapies hysteroscopy alone or combined with uterine artery embolization (group A) and direct laparoscopy alone or combined with laparoscopic uterine artery occlusion (group B) was selected based on the myometrial thickness between the gestational mass and the bladder. Results: The uteri of all 53 patients with CSPs were conserved; no conversion to laparotomy or blood transfusion was required. Uterine rupture occurred in one case during surgery in group $A$. The operative time in group $B$ was longer than group $A(42 \pm 18 \mathrm{vs} .80 \pm 33 \mathrm{~min} ; \mathrm{p}=0.022)$. The two groups were also similar with respect to other characteristics $(p>0.05)$. Conclusion: Myometrial thickness should be considered during the management of CSPs. Surgical approaches in the treatment of CSPs using 2-mm boundaries may yield an optimal clinical outcome.
\end{abstract}

Copyright $\odot 2013$ S. Karger AG, Basel

\section{KARGER}

(c) 2013 S. Karger AG, Basel

0378-7346/13/0763-0151\$38.00/0

E-Mail karger@karger.com

www.karger.com/goi

\section{Introduction}

Caesarean scar pregnancies (CSPs) have been reported as one of the rarest forms of ectopic pregnancy prior to 2005 [1]. Over the past 7 years, there has been a substantial increase in the number of CSPs because of the rising caesarean section rate and improvements in ultrasound diagnosis. There is no standard treatment for CSPs. Termination of pregnancy is recommended soon after confirmation of a CSP to avoid catastrophic complications, such as uterine rupture and heavy bleeding. Proposed interventions for CSPs include medical (feticide with systemic or local administration of methotrexate; MTX) [2] and surgical intervention (dilatation and curettage (D\&C), hysteroscopy, laparoscopy, laparotomy, or hysterectomy) [3]. Recently, uterine artery embolization (UAE) has been suggested as an alternative to systemic administration of MTX [4]. The aim of the present study was to describe our experience in the treatment of CSPs using a variety of approaches based on myometrial thickness, and to evaluate the safety and efficacy of these approaches in different clinical situations. 

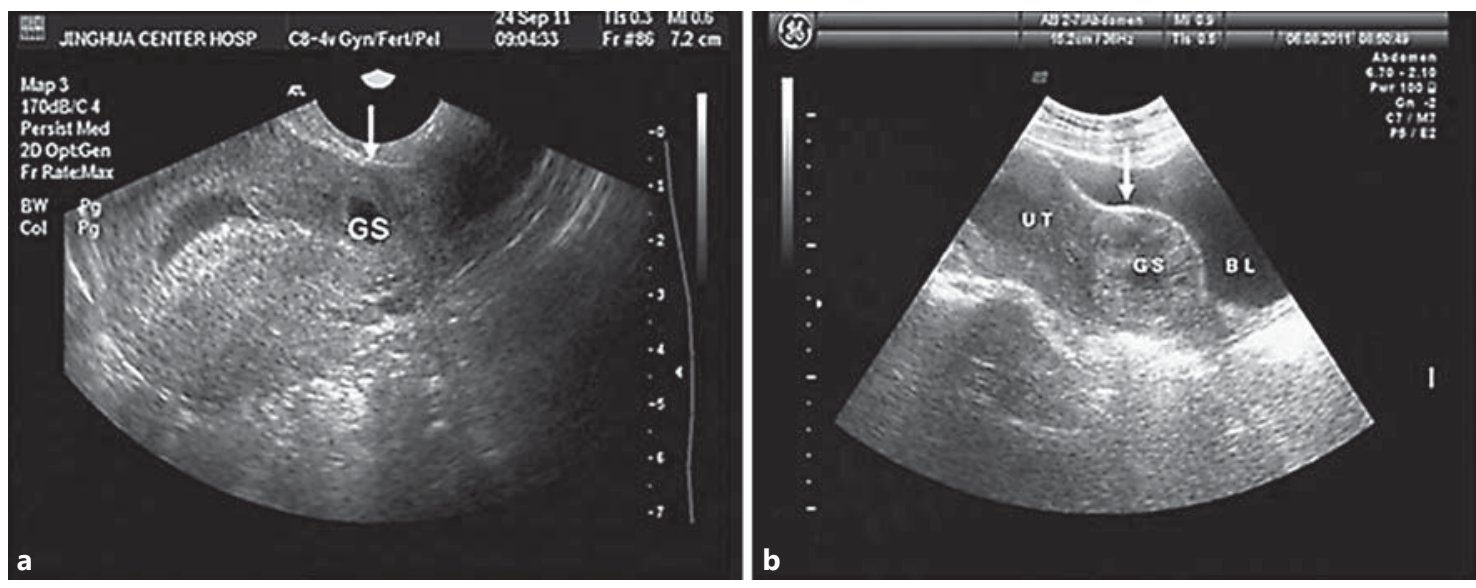

Fig. 1. a Transvaginal image. An ectopic sac is located in the lower segment of the anterior uterine wall, the myometrial thickness between the gestation mass and the bladder wall measuring $3 \mathrm{~mm}$. b Ultrasound showing a gestation mass deeply implanted at the site of a previous caesarean scar, there is no muscular coverage in this scar area.

\section{Materials and Methods}

\section{Patients}

A total of 53 women admitted to the hospital for treatment of CSPs between January 2009 and January 2012 were consecutively enrolled in the study. There were 3,890 deliveries and 2,348 caesarean sections carried out at our hospital during this period, and $60,000-70,000$ deliveries and 32,000-43,000 caesarean sections are performed each year in our whole region. The study protocol was approved by the Ethics Committee of Jinhua Municipal Central Hospital. Patients with ruptured uteri, severe bleeding, and liver or kidney disease were excluded. All of the selected women underwent removal of the retained products of conception. The participants were informed of the potential complications, benefits and alternatives of each approach, and written informed consent was also obtained. We emphasized the possibility of conversion to a laparotomy or hysterectomy if uncontrolled hemorrhage developed. Adverse effects, including fever, nausea and vomiting, stomatitis, alopecia, pelvic pain, and abnormal complete blood count or liver/renal function were recorded. Successful outcome was defined as normalization of serum $\beta$-hCG levels and resolution of CSP masses within 3 months, and a lack of severe complications, including ruptured uterus, massive hemorrhage (estimated blood loss $>1,000 \mathrm{ml}$ ), or hysterectomy.

\section{Diagnosis of CSP}

CSP was confirmed if the following criteria were met: a history of caesarean delivery, positive serum $\beta$-hCG level, and fulfillment of the ultrasound conditions [5] (an empty uterine cavity and empty cervical canal, development of the gestational sac/mass in the anterior part of the uterine isthmus, absence of a healthy myometrium between the bladder wall and the sac/mass, and color Doppler ultrasound demonstrating blood flow surrounding the gestational sac/mass). The distance between the gestational sac/mass and the bladder wall was measured with abdominal and transvaginal ultrasound by the same experienced specialist (fig. 1).

\section{Treatment Protocol}

MTX

All of the patients were administered systemic intramuscular MTX initially, a different dose of MTX (75-300 mg) and alternateday folinic acid rescue was required according to serum $\beta$-hCG levels and subtrophoblastic blood flow velocity. A local injection of MTX (20 mg) was added if the gestational sac was $\geq 2 \mathrm{~cm}$ with or without cardiac activity on transvaginal ultrasound. Transvaginal ultrasound, complete blood counts, and liver/renal function tests were determined weekly and $\beta$-hCG levels were obtained once a week for 2 weeks, then twice a week. Serum $\beta$-hCG decreasing more than half of the initial value before day 17 was defined as a good response to MTX. In these cases, we continued to wait until the serum $\beta$-hCG decreased to $<3,000 \mathrm{IU} / \mathrm{l}$, and the sub-trophoblastic blood flow velocity was nearly undetectable on transvaginal ultrasound. Direct hysteroscopy or laparoscopy was performed. If the serum $\beta$-hCG decreased by less than half on day 17 , failure of MTX treatment was considered and UAE or laparoscopic uterine artery occlusion (LUAO) was added to control bleeding prior to surgery (fig. 2).

\section{UAE}

Patients who had failed systemic MTX with a myometrial thickness $\geq 2 \mathrm{~mm}$ (group A) were given additional UAE preoperatively. UAE was performed by experienced radiologists; details of the technique have been described by Liang et al. [4]. Catheters were correctly placed and embolized with gelatin sponge particles (Hangzhou Alicon Medical Co., Ltd., Zhejiang, China; fig. 3). Occlusion of the distal ends of the uterine arteries bilaterally was confirmed by subtraction angiography. After $24 \mathrm{~h}$, patients underwent hysteroscopy.

\section{Hysteroscopy}

Hysteroscopy was performed in patients with a myometrial thickness $\geq 2 \mathrm{~mm}$ (group A). With the patient under general anesthesia, a Hegar dilator (up to number 10) was needed to dilate the cervix. An operative hysteroscope with a $10-\mathrm{mm}$ external diameter 


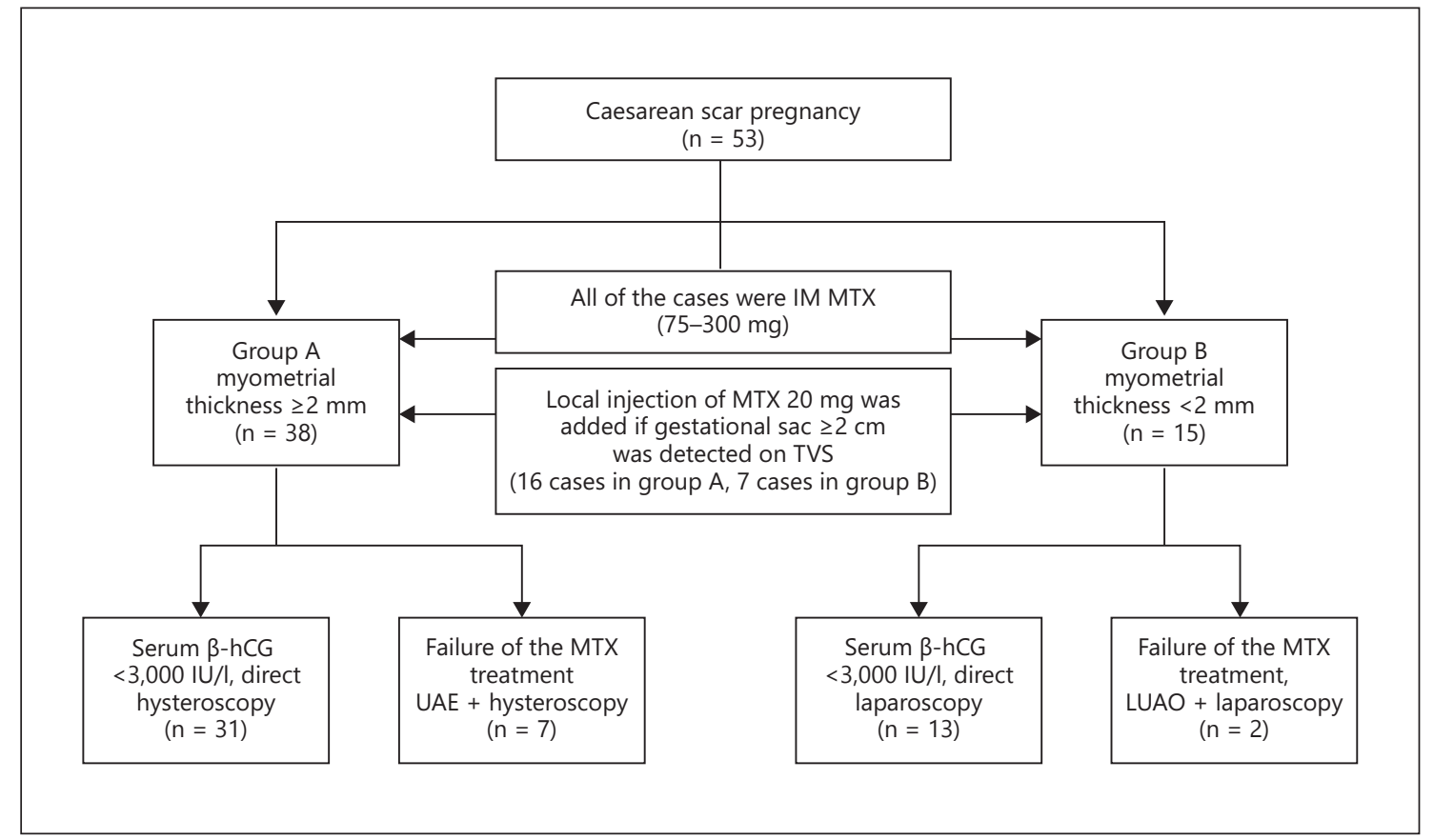

Fig. 2. Flow chart and criteria for CSP patients entering each arm.
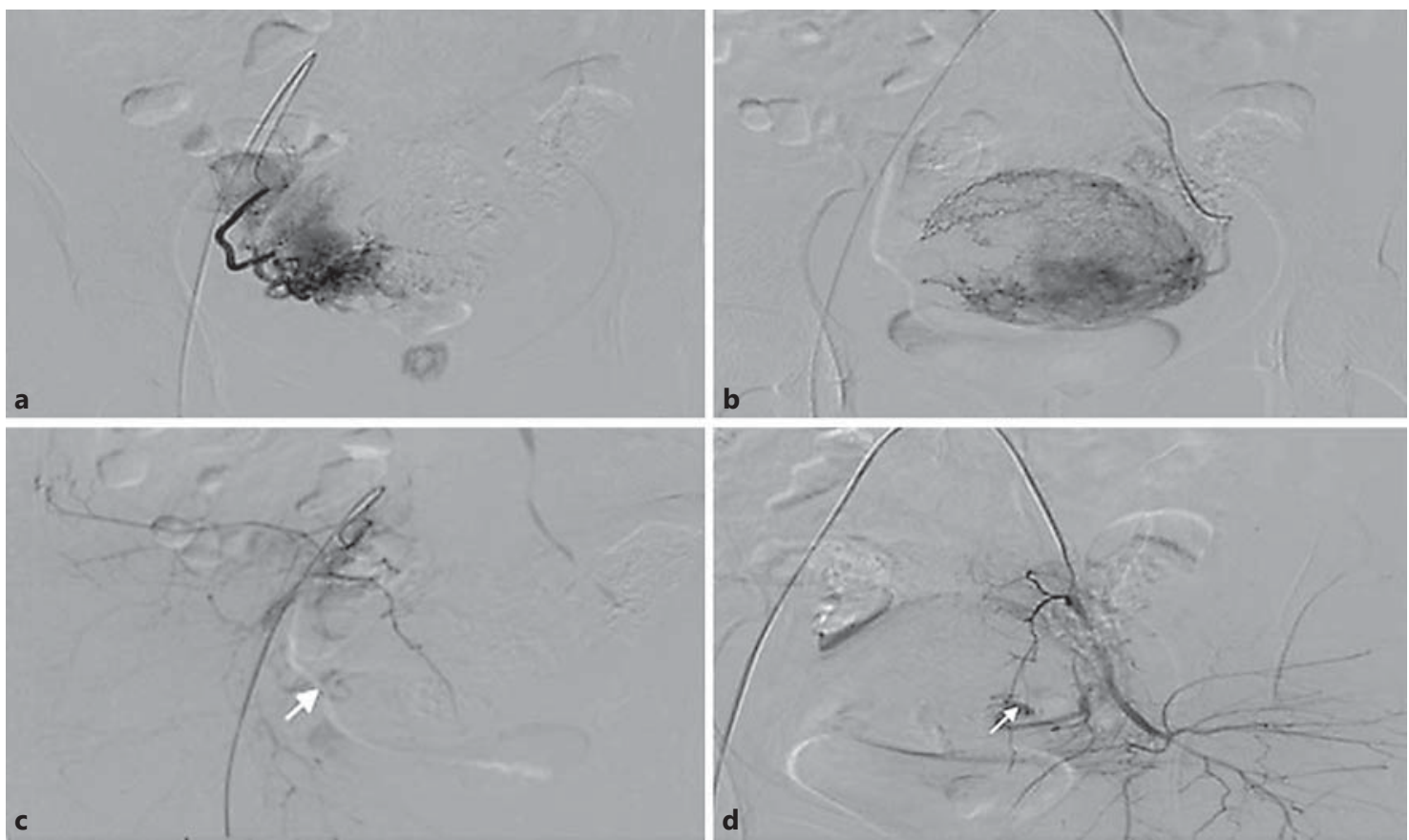

Fig. 3. Bilateral uterine angiography before $(\mathbf{a}, \mathbf{b})$ and after $(\mathbf{c}, \mathbf{d})$ UAE. Arrow indicates uterine artery. 

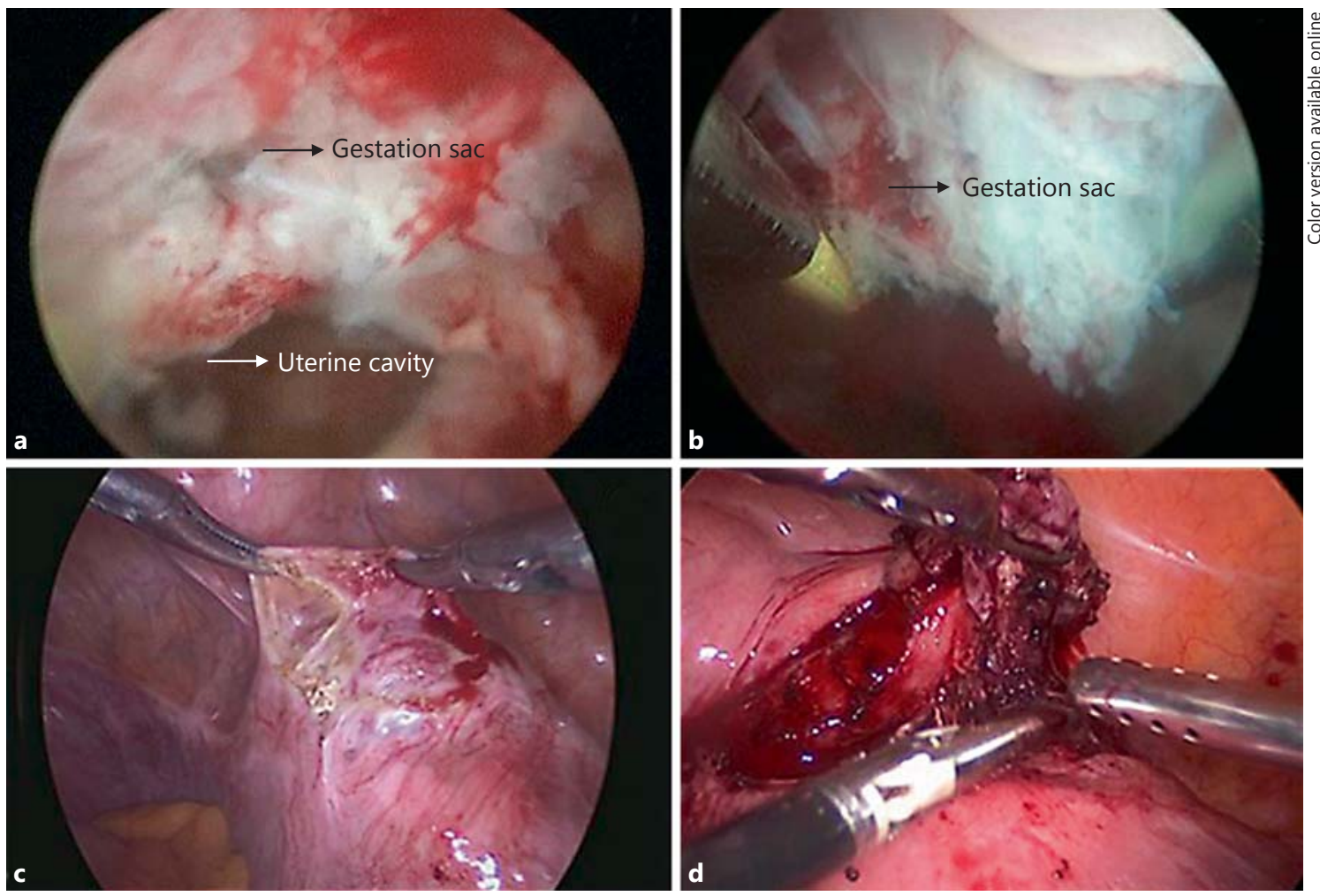

Fig. 4. a The gestation sac is located in the anterior part of the uterine defect wall, the uterine cavity is empty. b Hysteroscopy management of the CSP $(\mathbf{c}, \mathbf{d})$. A wedge resection is made under laparoscopy.

and a $4-\mathrm{mm} 30^{\circ}$ lens was placed inside the uterus, and normal saline was used to distend the uterus. Curettage and an electric loop were used to remove the gestational mass under direct vision; electrical coagulation and vasopressin was used to assure homeostasis (fig. 4).

\section{Laparoscopy}

Laparoscopy was used in patients with CSPs and a myometrial thickness $<2 \mathrm{~mm}$ (group B; fig. 4). A Foley catheter was placed in the bladder, and a uterine manipulator was placed within the uterine cavity. Four trocars were needed for the procedure. LUAO with bipolar coagulation was added to the patients who did not respond to MTX at the beginning of the procedure, and vasopressin was used if necessary. Successful artery dissection was performed as previously described [6]. Then, the bladder was dissected off the lower uterine segment of the uterus, exposing the bulging part of the gestational mass. This entire region was wedge-resected using unipolar scissors and grasping forceps, and the uterus was sutured in two layers.

\section{Statistical Analysis}

All statistical analyses were performed using SPSS (version 16.0; SPSS, Inc., Chicago, Ill., USA). Comparisons of quantitative data were performed using a t test or rank-sum test as appropriate. The categorical data were analyzed using a $\chi^{2}$ test. A two-tailed test was used and a $\mathrm{p}<0.05$ was considered statistically significant.

\section{Results}

In group A, 31 patients underwent hysteroscopy and 7 patients who failed MTX received UAE combined with hysteroscopy. In group B, 13 patients who underwent direct laparoscopy and 2 patients who failed MTX were managed with laparoscopy combined with LUAO. The baseline clinical characteristics of the subjects in the two groups are summarized in table 1 . There was no statistically significant difference between the two groups, including age, previous caesarean sections, abortions, the interval from the last caesarean delivery to the diagnosis of CSP, days of gestation, initial serum $\beta$-hCG level, gestational mass diameter, rates of cases with cardiac motion, and vaginal bleeding. All of the patients conceived spontaneously. Fifty-one patients had a prior diagnosis of CSP by ultrasound, and 4 patients underwent D\&C with a misdiagnosis of normal pregnancy in another hospital without massive bleeding. The myometrial thicknesses under ultrasonic surveillance were $0-5 \mathrm{~mm}$. 
Table 2. Comparison of clinical outcome after treatment in the two groups

\begin{tabular}{llll}
\hline Characteristic & Group A & Group B & p value \\
\hline Hyster/UAE + hysterectomy & $31 / 7$ & & \\
Lapar/LUAO + laparoscopy & & $13 / 2$ & \\
Success rate, n (\%) & $37(97.3)$ & $15(100)$ & 1.000 \\
Operative time, min & $42 \pm 18$ & $80 \pm 33$ & 0.022 \\
Operative blood loss, ml & $77 \pm 25$ & $95 \pm 32$ & 0.495 \\
Hospitalization, days & $19 \pm 8$ & $21 \pm 10$ & 0.876 \\
$\beta$-hCG to normal, days & $23 \pm 5$ & $21 \pm 6$ & 0.732 \\
Adverse effects, n (\%) & $8(21.1)$ & $2(13.3)$ & 0.706 \\
Resolution of CSP mass, days & $63 \pm 21$ & $70 \pm 24$ & 0.693 \\
\hline
\end{tabular}

Group A presenting as patients with myometrial thickness $\geq 2 \mathrm{~mm}$ and group $\mathrm{B}$ thoses with myometrial thickness $<2 \mathrm{~mm}$ patients. All cases were administered MTX initially.

Table 1. Baseline clinical characteristics of patients at diagnosis in the two groups

\begin{tabular}{lccc}
\hline Characteristic & Group A & Group B & $\begin{array}{l}\mathrm{p} \\
\text { value }\end{array}$ \\
\hline $\begin{array}{l}\text { Hyster/UAE + } \\
\quad \text { hysterectomy }\end{array}$ & $31 / 7$ & & \\
Lapar/LUAO + & & & \\
$\quad$ laparoscopy & & $13 / 2$ & \\
Age, years & $31.2 \pm 4.8$ & $33.9 \pm 5.5$ & 0.596 \\
CS (times) & $1.2 \pm 0.3$ & $1.1 \pm 0.2$ & 0.876 \\
Number of abortions & $2.2 \pm 1.3$ & $2.1 \pm 1.1$ & 0.775 \\
Interval from last CS, & & & \\
$\quad$ years & $5.2 \pm 3.5$ & $6.7 \pm 4.3$ & 0.379 \\
Gestational age, days & $60.7 \pm 14.5$ & $62.2 \pm 12.3$ & 0.747 \\
Initial $\beta$-hCG, IU/1 & $35,070 \pm 18,750.9$ & $40,208 \pm 23,200$ & 0.378 \\
Fetal heart beat, $\mathrm{n}(\%)$ & $8(21.1)$ & $1(6.7)$ & 0.418 \\
Gestational mass & & & \\
$\quad$ diameter, cm & $3.2 \pm 1.8$ & $4.0 \pm 2.3$ & 0.654 \\
Vaginal bleeding, & & & \\
$\quad \mathrm{n}(\%)$ & $16(42.1)$ & $5(33.3)$ & 0.556 \\
\hline
\end{tabular}

Group A presenting as patients with myometrial thickness $\geq 2 \mathrm{~mm}$ and group B as those with myometrial thickness $<2 \mathrm{~mm}$ patients. All cases were administered MTX initially.

The clinical outcomes after treatment are shown in table 2. As expected, the operative time in group B was longer than in group A ( $42 \pm 18$ vs. $80 \pm 33 \mathrm{~min} ; \mathrm{p}=0.022)$. The two groups were similar with respect to operative blood loss, length of hospital stay, resolution time of the CSP mass, and serum $\beta$-hCG level. In group A, uterus rupture occurred in 1 patient intraoperatively (UAE combined with hysteroscopy), and the uterine cleft was immediately sutured under laparoscopy. In group A, 3 patients had a mild increase in liver enzymes after MTX therapy, 4 had fevers with temperatures of $38-39.5^{\circ} \mathrm{C}$, and 1 complained of moderate pelvic pain after management with UAE. In group B, 1 patient had a mild increase in liver enzymes and 1 had mild vomiting after MTX therapy. There were no conversions to laparotomy, no blood transfusions, and no hysterectomies in either group. After discharge, regular follow-up was recommended, the serum $\beta$-hCG levels normalized within 1 month, and the resolution of CSP masses was confirmed by ultrasonography within 3 months. 3 of 7 patients treated with UAE attempted to become pregnant. The first patient gave birth to a healthy infant by caesarean section at 37 weeks' gestation. The second patient had a repeat scar pregnancy in the same year and was treated with laparoscopic gestational sac excision and re-approximation of the caesarean section defect. The third patient had a spontaneous abortion the year previously and has not achieved pregnancy to date.

\section{Discussion}

A CSP is a serious type of ectopic pregnancy, the optimal therapy of which is still unclear due to its rarity. The relatively higher prevalence in our area is mainly explained by the high caesarean section rates. In fact, therapeutic options for CSPs have been focused on uterine preservation. Medical treatment alone has been reported to successfully cure CSP in most cases [7]; however, this treatment option requires considerable patience from both the patients and doctors because the resolution time of the CSP mass may be as long as 177 days $[7,8]$. Wang et al. [9] analyzed 50 patients who were treated with MTX followed by D\&C and compared them with 20 patients who were treated with MTX only. They showed that the combined treatment resulted in a more favorable effect [9]. Surgical removal of the gestational mass was also recommended by Wang et al. [10] and Ash et al. [11], feticide or uterine artery occlusion is performed pre-operatively to avoid massive hemorrhage. Vial et al. [12] were the first to describe two different ultrasonographic patterns: surface implantation, and deep implantation of the amniotic sac in which the myometrial thickness between the mass and the bladder wall to $2 \mathrm{~mm}$ was considered deep implantation $[13,14]$. In these cases, the trophoblastic tissue is extremely difficult to remove by curettage without perforating the myometrium $[15,16]$. Even when an intra- 
uterine approach succeeds, a persistent uterine scar dehiscence or thin myometrium will potentially affect future pregnancies [16-18]. Some authors advocated D\&C under ultrasound guidance in cases in which the myometrial thickness is $>3.5 \mathrm{~mm}[19,20]$ because the optimal cut-off values for predicting uterine scar defects and the risk of uterine rupture near term range from 1.4 to 2.0 $\mathrm{mm}$ for the myometrial layer [21, 22], and a myometrial thickness of $>1.5 \mathrm{~mm}$ is considered not to be at risk for uterine dehiscence [23]. We attempted to use $2 \mathrm{~mm}$ as the threshold for myometrial thickness between the gestational mass and the bladder with respect to intrauterine or abdominal approach for therapy to determine the feasibility of the different methods in different clinical conditions. The advantages and disadvantages of various treatments were also discussed.

MTX is used as a primary therapy to perform feticide, and is suitable for both patterns of implantation in clinically stable women prior to surgical intervention. MTX can help inhibit trophoblast proliferation, resolve subtrophoblastic blood flow, eradicate chorionic villi, and decrease serum $\beta$-hCG levels. Thus, removing the entire CSP mass is facilitated with controlled uterine bleeding using an intrauterine or abdominal approach. Some gestational sacs $<2.5 \mathrm{~cm}$ in size can even detach from the scar section and spontaneously expel after MTX treatment in our study, expectant management seems advisable at least in these patients. However, the patients cannot be ensured when the CSP mass completely resolve and it definitely could prolong bleeding and thus interfere with their daily lives. Moreover, during the follow-up period, secondary heavy bleeding and infection may occur, so the patients still demanded D\&C instead of expectant management. In the present study, 44 of 53 patients who received MTX as primary therapy were successfully treated, 4 patients had mild increases in liver enzymes and 1 had mild vomiting. These symptoms resolved soon after symptomatic treatment.

UAE was performed in patients who failed MTX treatment; with the flow of uterine blood blocked, the patients could undergo hysteroscopy after $24 \mathrm{~h}$. Because sponge particles achieve complete vascular embolization, UAE is more effective and reliable than LUAO, but UAE is associated with a greater risk of intrauterine necrosis [24]. The long-term reproductive outcomes of UAE and LUAO are uncertain at present. It has been reported that both UAE and LUAO might impair reproductive ability [25-27], while Holub et al. [28] reported that patients who undergo LUAO have more favorable pregnancy outcomes than patients who undergo UAE. Our study cannot demonstrate the relationship between UAE and reproductive ability due to a too small sample size, but UAE tends to bring more complications. As shown in our study, of the 7 patients managed with UAE, 4 developed fevers and 1 complained of moderate pelvic pain. UAE has other limitations, such as increased medical costs and technical demands. Thus, UAE should not be a first-line therapeutic option; LUAO is preferred in clinically stable women who undergo an abdominal approach for CSP.

In 2005, Wang et al. [29] described a successful treatment for CSP by hysteroscopy; with the direct visualization of the CSP, hysteroscopy helps establishment a definitive diagnosis, remove the gestational sac/mass and coagulation the blood vessels more accurately. However, if the anterior uterine wall is too thin, hysteroscopy may perforate the CSP and injure the bladder. Consequently, myometrial thickness should be considered during the management of CSP. In our study, 38 patients with a myometrial thickness $\geq 2 \mathrm{~mm}$ adopted the intrauterine approach, and 1 patient with a myometrial thickness of $2 \mathrm{~mm}$ failed. The reason for the failed intrauterine treatment approach may have involved the large gestational mass $(3.8 \times 4.5 \mathrm{~cm})$ protruding towards the abdominal cavity with firm adhesion to the caesarean scar site or invasion of trophoblastic cells into the caesarean scar, which caused the intrauterine procedure to be more difficult. Our experience suggests that the intrauterine approach is safe in cases with a myometrial thickness $\geq 2 \mathrm{~mm}$ and a gestational mass $<3 \mathrm{~cm}$ in size or a gestational sac protruding towards the cervicoisthmic space or the uterine cavity.

If the muscular layer in the scar area is too thin and some parts of the gestational mass do not have muscular coverage, laparoscopy is the preferred treatment option. In our study, LUAO with bipolar coagulation instead of UAE was used to control bleeding in those patients who failed MTX treatment. Although this study showed that the operative time in group B was longer than group A, there was no statistically significant difference between the two groups with respect to other characteristics. Furthermore, surgery completely removes the pregnancy and repairs the scar defect, which will cause a predisposition to another ectopic implantation [30, 31]. Godin et al. [5] and Deans and Abbott [32] also demonstrated that strengthening the uterine wall of the scar area is necessary, especially in those patients who desire fertility.

This study retrospectively investigated the management of 53 patients with CSPs using $2 \mathrm{~mm}$ as a threshold 
myometrial thickness, and treatment of the CSP using an intrauterine or abdominal approach with a high success rate and rapid recovery. However, the sample size was small and a larger study is required to confirm our conclusion.

\section{Disclosure Statement}

The authors have no conflicts of interest.

\section{References}

1 Graesslin O, Dedecker F Jr, Quereux C, Gabriel R: Conservative treatment of ectopic pregnancy in a cesarean scar. Obstet Gynecol 2005; 105:869-871.

-2 Halperin R, Vaknin Z, Schneider D, Yaron M, Herman A: Conservative management of ectopic pregnancy with fetal cardiac activity by combined local (sonographically guided) and systemic injection of methotrexate. Gynecol Obstet Invest 2003;56:148-151.

3 Rotas MA, Haberman S, Levgur M: Cesarean scar ectopic pregnancies: etiology, diagnosis, and management. Obstet Gynecol 2006;107: 1373-1381.

4 Li C, Li C, Feng D, Jia C, Liu B, Zhan X: Transcatheter arterial chemoembolization versus systemic methotrexate for the management of cesarean scar pregnancy. Int J Gynaecol Obstet 2011;113:178-182.

5 Godin P-A, Bassil S, Donnez J: An ectopic pregnancy developing in a previous caesarean section scar. Fertil Steril 1997;67:398-400.

6 Holub Z, Eim J, Jabor A, Hendl J, Lukac J, Kliment L: Complication and myoma recurrence after laparoscopic uterine occlusion for symptomatic myomas. J Obstet Gynaecol Res 2006; 32:55-62.

-7 Timor-Tritsch IE, Monteagudo A, Santos R, Tsymbal T, Pineda G, Arslan AA: The diagnosis, treatment, and follow-up of cesarean scar pregnancy. Am J Obstet Gynecol 2012; 207:44.e1-e13.

8 Seow K-M, Huang L-W, Lin YH, Yan-Sheng Lin M, Hwang J-L: Cesarean scar pregnancy: issues in management. Ultrasound Obstet Gynecol 2004;23:247-253.

-9 Wang JH, Xu KH, Lin J, Xu JY, Wu RJ: Methotrexate therapy for cesarean section scar pregnancy with and without suction curettage. Fertil Steril 2009;92:1208-1213.

10 Wang CJ, Chao AS, Yuen LT, Wang CW, Soong YK, Lee CL: Endoscopic management of cesarean scar pregnancy. Fertil Steril 2006; 85:494.e1-e4.

11 Ash A, Smith A, Maxwell D: Caesarean scar pregnancy. BJOG 2007;114:253-263.

12 Vial Y, Petignat P, Hohlfeld P: Pregnancy in a cesarean scar. Ultrasound Obstet Gynecol 2000; $16: 592-593$.
13 Weimin W, Wenqing L: Effect of early pregnancy on a previous lower segment cesarean section scar. Int J Gynecol Obstet 2002;77: 201-207.

14 Tinelli A, Tinelli R, Malvasi A: Laparoscopic management of cervical-isthmic pregnancy: a proposal method. Fertil Steril 2009;92:829 e3-e6.

15 Chang Y, Kay N, Chen YH, Chen HS, Tsai EM: Resectoscopic treatment of ectopic pregnancy in previous cesarean delivery scar defect with vasopressin injection. Fertil Steril 2011;96:80-82

16 Fylstra DL: Ectopic pregnancy within a cesarean scar: a review. Obstet Gynecol Surv 2002; 57:537-543.

17 Haimov-Kochman R, Sciaky-Tamir Y, Yanai $\mathrm{N}$, Yagel S: Conservative management of two ectopic pregnancies implanted in previous uterine scars. Ultrasound Obstet Gynecol 2002;19:616-619.

18 Osser OV, Jokubkiene L, Valentin L: High prevalence of defects in Cesarean section scars at transvaginal ultrasound examination. Ultrasound Obstet Gynecol 2009;34:90-97.

19 Arslan M, Pata O, Dilek TU, Aktas A, Aban M, Dilek S: Treatment of viable cesarean scar ectopic pregnancy with suction curettage. Int J Gynecol Obstet 2005;89:163-166.

20 Wang C-B, Tseng C-J: Primary evacuation therapy for Cesarean scar pregnancy: three new cases and review. Ultrasound Obstet Gynecol 2006;27:222-226.

21 Jastrow N, Chaillet N, Roberge S, Morency AM, Lacasse Y, Bujold E: Sonographic lower uterine segment thickness and risk of uterine scar defect: a systematic review. J Obstet Gynaecol Can 2010;32:321-327.

-22 Marasinghe JP, Senanayake H, Randeniya C, Seneviratne HR, Arambepola C, Devlieger R Comparison of transabdominal versus transvaginal ultrasound to measure thickness of the lower uterine segment at term. Int J Gynaecol Obstet 2009;107:140-142.

23 Gizzo S, Zambon A, Saccardi C, Patrelli TS, Di Gangi S, Carrozzini M, Bertocco A, Capobianco G, D'Antona D, Nardelli GB: Effective anatomical and functional status of the lower uterine segment at term: estimating the risk of uterine dehiscence by ultrasound. Fertil Steril 2013;99:496-501.
24 Kuzel D, Mara M, Horak P, Kubinova K, Maskova J, Dundr P, Cindr J: Comparative outcomes of hysteroscopic examinations performed after uterine artery embolization or laparoscopic uterine artery occlusion to treat leiomyomas. Fertil Steril 2011; 95:2143-2145.

25 Mara M, Maskova J, Fucikova Z, Kuzel D, Belsan T, Sosna O: Midterm clinical and first reproductive results of a randomized controlled trial comparing uterine fibroid embolization and myomectomy. Cardiovasc Intervent Radiol 2008;31:73-85.

-26 Hardeman S, Decroisette E, Marin B, Vincelot A, Aubard Y, Pouquet M, Maubon A: Fertility after embolization of the uterine arteries to treat obstetrical hemorrhage. A review of 53 cases. Fertil Steril 2010;94:2574-2579.

27 Chen YJ, Wang PH, Yuan CC, Yen YK, Yang MJ, Ng HT, Chang SP, Liu WM: Pregnancy following treatment of symptomatic myomas with laparoscopic bipolar coagulation of uterine vessels. Hum Reprod 2003;18:10771081.

28 Holub Z, Mara M, Kuzel D, Jabor A, Maskova J, Eim J: Pregnancy outcomes after uterine artery occlusion: prospective multicentric study. Fertil Steril 2008;90:1886-1891.

29 Wang C-J, Yuen L-T, Chao A-S, Lee C-L, Yen C-F, Soong Y-K: Caesarean scar pregnancy successfully treated by operative hysteroscopy and suction curettage. BJOG 2005;112:839840

30 Ben Nagi J, Ofili-Yebovi D, Sawyer E, Aplin J, Jurkovic D: Successful treatment of a recurrent caesarean scar ectopic pregnancy by surgical repair of the uterine defect (letters to the editor). Ultrasound Obstet Gynecol 2006;28: 855-857.

31 Hasegawa J, Ichizuka K, Matsuoka R, Otsuki K, Sekizawa A, Okai T: Limitations of conservative treatment for repeat cesarean scar pregnancy. Ultrasound Obstet Gynecol 2005;25: 310-311.

32 Deans R, Abbott J: Hysteroscopic management of cesarean scar ectopic pregnancy. Fertil Steril 2010;93:1735-1740. 